\title{
Review of: "Gradient association between pulmonary tuberculosis and diabetes mellitus among households with a tuberculosis case: a contact tracing-based study"
}

\section{Subhakar Kandi}

Potential competing interests: The author(s) declared that no potential competing interests exist.

The bidirectional association between tuberculosis (TB) and diabetes mellitus (DM) is currently one of the major concerns for clinicians, as DM affects the disease presentation and clinical outcome of TB and vice versa. The interest in diabetes mellitus and tuberculosis is mounting rapidly and it promises to be an exciting time for researchers involved in the study of dual diseases. Overall this is a brilliant article and the observations in the studies presented add emphasis to the underlying need for Bidirectional screening of Close contacts of TB for diabetes mellitus and diabetics for Tuberculosis. Here are a few suggestions on potential enhancements to the article:

1. The authors used TB cultures for diagnosis of Pulmonary Tuberculosis in household contacts of TB patients which could be long diagnostic process. Using rapid molecular tests like CBNAAT instead could have allowed for quicker diagnosis and bigger sample sizes being tested

2. Some of the subjects in the study are culture negative PTB cases but the author(s) missed specifying the diagnostic tool used for them

3. The authors also haven't mentioned the criteria for labelling patients as pre-diabetic and diabetic. A set of guidelines or standards (by diabetes Association for example) used for labelling the data could provide more weight to the findings

4. The conclusion that Age $>=35$ years, excessive edible oil intake and DM family history are significant predictors of diabetes could've been backed by including data such as a table presenting how incidence varies by each age bucket, providing more objective information on what values would constitute excessive oil intake, p-values, confidence intervals etc. 